\title{
Goal-oriented therapy in paediatric pulmonary arterial hypertension: are we ready?
}

\author{
Maurice Beghetti
}

Affiliation: Pediatric Cardiology Unit, Children's University Hospital, Geneva, Switzerland.

Correspondence: Maurice Beghetti, Pediatric Cardiology Unit, Children's University Hospital, 6 rue Willy Donzé, 1211 Geneva 14, Switzerland. E-mail: maurice.beghettiahcuge.ch

0

@ERSpublications

A treatment goal approach is a major progress that should allow for an improved outcome in pediatric PAH http://ow.ly/C63da

Pulmonary arterial hypertension (PAH) is still a fatal disease, affecting patients of all ages including children [1]. In the last 20 years, significant progress has been achieved in the understanding of the disease, and in particular, in the medical approach, with the introduction of new therapies. Goal-oriented strategy represents a significant progress in PAH management and has emerged as the best treatment approach for PAH in adults [2]. Indeed, it is considered as part of the daily practice and should lead to an increase in therapy when goals are not met. In the European Society of Cardiology/European Respiratory Society guidelines for diagnosis and treatment or pulmonary hypertension treatment, goals were suggested $[3,4]$. NICKEL et al. [5] described their own, local, approach towards adults with PAH and showed a significant improvement in survival when a goal-oriented strategy was applied [5]. The concept is based on defining markers of the disease and follows their changes systematically, and then adapting therapy to these changes. To be successful, it requires the identification of the markers along with the changes that correlate to an improved outcome. At the last world symposium on pulmonary hypertension, held in Nice (France), a specific task force was dedicated to the treatment goals of pulmonary hypertension, and all potential treatment goals were discussed [2]. This approach is thought to be valid for all groups of patients included in the group 1 of the clinical classification for pulmonary hypertension. But are treatment goals similar for all groups of PAH patients (group 1 of the clinical classification)? A specific discussion raised the important question: are treatment goals different in different subgroups of pulmonary arterial hypertension? This is a key question as the PAH group is a heterogeneous group of patients. In particular, the paediatric group can be considered as a specific group and at the last world symposium it benefited from a specific task force [1], and part of the published proceedings of this group related to treatment goals.

However, a recurrent problem with paediatric PAH is the lack of robust studies allowing for strong conclusions in this population. Recent data coming from large registries have highlighted the difference in epidemiology and aetiology compared to the adult population [6-8] as well as the diagnostic approach in paediatric pulmonary hypertension [9]. The paediatric part of the REVEAL registry (Registry to Evaluate Early And Long-term PAH Disease Management) [7], suggested some risk factors, but they cannot be used currently as treatment goals. Similarly, some other single, centre studies have described other risk factors.

There is an urgent need to define a goal-oriented therapy in children with PAH [10] and in this issue of the European Respiratory Journal, PLOEgSTRA et al. [11] have to be congratulated on presenting their important data. This study suggests some treatment goals for the paediatric PAH population. Although only 62 patients were recruited for this analysis, several criteria allows for robustness of the data, the single centre

Received: Sept 052014 | Accepted: Sept 052014

Conflict of interest: Disclosures can be found alongside the online version of this article at erj.ersjournals.com

Copyright @ERS 2014 
design and, in the Netherlands, this centre is the national referral centre for paediatric pulmonary hypertension, allowing for a consistent and systematic approach for all patients. In addition all analysed patients were treatment naïve, which is one of the most important aspects for this analyses approach. However, we may just notice that the recruitment covers a period of 13 years and this may induce some bias. This increases the difficulty, within paediatric PAH research, in collecting robust data. Hence ongoing large registries, such as the TOPP registry (registry for Tracking practice and Outcome in Pediatric Pulmonary hypertension), will hopefully confirm the pioneering work by PloEgSTRA et al. [11].

As highlighted by Ploegstra et al. [11], one must be cautious in defining treatment goals, as this is different from risk factors. If risk factors at diagnosis have been recognised in the paediatric population, we are still lacking validation of potential treatment goals. Treatment goals are variables that show changes in correlation with the treatment, and correlate with outcome.

Functional class [11, 12]; haemodynamic parameters (ratio of mean pulmonary arterial to systemic artery pressure [12], right atrial pressure $>10 \mathrm{mmHg}$ and pulmonary vascular resistance index (PVRI) $>20$ Wood units $\cdot \mathrm{m}^{-1}[2,7,13]$; brain natriuretic peptide $(\mathrm{BNP}) / \mathrm{N}$-terminal pro-BNP $(\mathrm{BNP} / \mathrm{NT}-$ proBNP) $[12,14,15]$ and echo parameters (systolic to diastolic duration ratio [16]; right to left ventricular diameter [17]; and tricuspid plane annular excursion (TAPSE) [11], have all been reported to correlate with outcome when measured at diagnosis. Some of these markers have indeed been used to elaborate the paediatric determinants of risk [1]. However, changes in these variables are not currently recognised as treatment goals, as no data have so far confirmed that their changes correlate with outcome. PLOEGSTRA et al. [11] recognise functional class, TAPSE and NT-proBNP as treatment goals.

Functional class has been recognised as a treatment goal in adults [2], and in addition, despite consideration about the definition of the functional class in children [18], multiple reports confirm the validity of functional class as a risk factor in paediatrics $[7,11,12]$. Reaching functional class II, for adults, was found to have led to a better outcome. Ploegstra et al. [11] report that improving from functional class IV is a predictor for a better outcome; however, they could not show the same results in children as had been previously observed in adults, i.e. functional class III appears, from their study, to be the goal for children as opposed to functional class II, which is the goal for adults. However, it is clearly mentioned that the results should be taken with caution, due to the lack of power by the study cohort. It is probable that data from a larger group of patients could show that functional class II is also the goal for paediatric patients, with functional class III not being the ideal.

Several, single centre, retrospective, paediatric studies have shown that BNP/NT-proBNP is a biomarker of prognostic value, when measured at diagnosis $[12,14,15]$. Again, Ploegstra et al. [11] could prove that a decrease in NT-proBNP, in the follow-up, is a predictor and fulfils the criteria for a treatment goal in children. They report a threshold of $<1200 \mathrm{ng} \cdot \mathrm{L}^{-1}$ as a potential goal. A word of caution should always be raised when the threshold for NT-proBNP is reported, as sometimes different methods of measurements are used in different laboratories and thresholds may vary. Taking this into account, this biomarker seems to be of major interest during the follow-up of paediatric PAH patients.

There is a major interest in developing echocardiographic variables as risk factors and/or potential treatment goals, as this is a noninvasive technique that can easily be performed in paediatric patients. In addition, there are some concerns regarding the use of repeated invasive haemodynamic assessments in children, due to the potential risk of cardiac catheterisation in this population, even if haemodynamics remain important prognostic parameters. [9]. TAPSE is an excellent candidate, as it correlates with right ventricular function and is supported by several adult studies [19-21]. However, there are still some controversies about the use of TAPSE in adults as a treatment goal and some experts challenge the published results, arguing that it may not be validated in multivariate analysis and, in addition, it may be misleading in the presence of tricuspid regurgitation or after congenital heart disease repair. These data are still not published, but should be available soon. However, the study by Ploegstra et al. [11], confirms the possibility to use TAPSE changes as a treatment goal in children, by giving a threshold $(>12 \mathrm{~mm})$ that seems to be correlated with improved outcome. We have again to be cautious with thresholds, as TAPSE in children should probably be adapted to size. Normal TAPSE in a newborn cannot be the same as in an adolescent. Ploegstra et al. [11] raises this particular problem and the Z-score may be a better approach.

We are now close to developing a goal-oriented approach in children with these new data. We should still confirm these results in larger cohorts of patients, but it is promising and similar results are expected form uncontrolled single or multicentre studies or registries (TOPP or REVEAL) very soon. In addition, controlled and uncontrolled studies are indeed currently being performed on new drug treatments in children and have included other potential goals (echocardiography, haemodynamics, biomarkers, functional class etc.) as secondary endpoints. Interestingly, so far, exercise capacity has not been recognised 
as a risk factor or a treatment goal in paediatrics compared to adults. This may be due to the difficulty to perform and reproduce exercise testing; either 6-min walk test or cardiopulmonary exercise in children. So far controversial results are published [22-24], but we may soon have data supporting its use as a potential goal in paediatric patient over 7-8 years of age. Other studies are analysing multiple factors that can be measured during cardiopulmonary exercise that may be related to outcome.

So the answer to the question, "are we ready to develop a treatment goal strategy in paediatric PAH?" is probably yes. This is, indeed, what has been undertaken in most referral expert centres, but so far without data confirming its validity. Ploegstra et al. [11] clearly paved the way for this approach and we must now validate these data in large cohorts and studies. We need to prove that these goals are of value for all age groups and aetiologies of PAH in paediatrics and also try to validate some other goals to further improve care. This is underway in several studies.

We cannot conclude without raising a specific aspect of treatment for paediatric PAH that could indeed challenge the treatment goal approach. In adults, the treatment goal is standard practice, and prompts the increase in therapy, double or triple combination therapy. The problem we currently have in children is that we have very few drugs, if any in some countries, which have been approved for treatment. Several studies are indeed currently ongoing with the treatment used in adults, to confirm efficacy and safety as well as the dose that should be used. Hopefully, these studies should solve this problem and lead to drug approval. However, the current available data, describing the use in clinical practice of these drugs in paediatrics, are in favour of their efficacy and safety and allows following a treatment goal approach.

The paediatric PAH community has recently made great progress in improving the diagnosis and treatment of PAH, but we still have a long way to go before finding a cure. Until such a time, optimising the available treatments is the best way of providing the most effective care for these difficult and fragile patients, and a treatment goal is progress that should allow for an improved outcome.

\section{References}

1 Ivy DD, Abman SH, Barst RJ, et al. Pediatric pulmonary hypertension. J Am Coll Cardiol 2013; 62: Suppl., D117-D126.

2 McLaughlin VV, Gaine SP, Howard LS, et al. Treatment goals of pulmonary hypertension. J Am Coll Cardiol 2013; 62: Suppl., D73-D81.

3 Galiè N, Hoeper MM, Humbert M, et al. Guidelines for the diagnosis and treatment of pulmonary hypertension. Eur Respir J 2009; 34: 1219-1263.

4 Galiè N, Hoeper MM, Humbert M, et al. Guidelines for the diagnosis and treatment of pulmonary hypertension. Eur Heart J 2009; 30: 2493-2537.

5 Nickel N, Golpon H, Greer M, et al. The prognostic impact of follow-up assessments in patients with idiopathic pulmonary arterial hypertension. Eur Respir J 2012; 39: 589-596.

6 Berger RMF, Beghetti M, Humpl T, et al. Clinical features of paediatric pulmonary hypertension: a registry study. Lancet 2012; 379: 537-546.

7 Barst RJ, McGoon MD, Elliott CG, et al. Survival in childhood pulmonary arterial hypertension: insights from the registry to evaluate early and long-term pulmonary arterial hypertension disease management. Circulation 2012; 125: $113-122$.

8 Lévy M, Celermajer D, Szezepanski I, et al. Do tertiary paediatric hospitals deal with the same spectrum of paediatric pulmonary hypertension as multicentre registries? Eur Respir J 2013; 41: 236-239.

9 Beghetti M, Berger RMF, Schulze-Neick I, et al. Diagnostic evaluation of paediatric pulmonary hypertension in current clinical practice. Eur Respir J 2013; 42: 689-700.

10 Berger RMF. Pulmonary hypertension: smaller kids, smaller steps. Lancet Respir Med 2014; 2: 348-350.

11 Ploegstra MJ, Douwes JM, Roofthooft MTR, et al. Identification of treatment goals in paediatric pulmonary arterial hypertension. Eur Respir J 2014; 44: 1616-1626.

12 van Loon RL, Roofthooft MTR, Delhaas T, et al. Outcome of pediatric patients with pulmonary arterial hypertension in the era of new medical therapies. Am J Cardiol 2010; 106: 117-124.

13 Ivy DD, Rosenzweig EB, Lemarié JC, et al. Long-term outcomes in children with pulmonary arterial hypertension treated with bosentan in real-world clinical settings. Am J Cardiol 2010; 106: 1332-1338.

14 Lammers AE, Hislop AA, Haworth SG. Prognostic value of B-type natriuretic peptide in children with pulmonary hypertension. Int J Cardiol 2009; 135: 21-26.

15 Takatsuki S, Wagner BD, Ivy DD. B-type natriuretic peptide and amino-terminal pro-B-type natriuretic peptide in pediatric patients with pulmonary arterial hypertension. Congenit Heart Dis 2012; 7: 259-267.

16 Alkon J, Humpl T, Manlhiot C, et al. Usefulness of the right ventricular systolic to diastolic duration ratio to predict functional capacity and survival in children with pulmonary arterial hypertension. Am J Cardiol 2010; 106: 430-436.

17 Jone PN, Hinzman J, Wagner BD, et al. Right ventricular to left ventricular diameter ratio at end-systole in evaluating outcomes in children with pulmonary hypertension. J Am Soc Echocardiogr 2014; 27: 172-178.

18 Lammers AE, Adatia I, Cerro MJ, et al. Functional classification of pulmonary hypertension in children: report from the PVRI pediatric taskforce, Panama 2011. Pulm Circ 2011; 1: 280-285.

19 Forfia PR, Fisher MR, Mathai SC, et al. Tricuspid annular displacement predicts survival in pulmonary hypertension. Am J Respir Crit Care Med 2006; 174: 1034-1041.

20 Mathai SC, Sibley CT, Forfia PR, et al. Tricuspid annular plane systolic excursion is a robust outcome measure in systemic sclerosis-associated pulmonary arterial hypertension. J Rheumatol 2011; 38: 2410-2418. 
21 Mercer-Rosa L, Parnell A, Forfia PR, et al. Tricuspid annular plane systolic excursion in the assessment of right ventricular function in children and adolescents after repair of tetralogy of Fallot. J Am Soc Echocardiogr 2013; 26: $1322-1329$.

22 Lammers AE, Diller GP, Odendaal D, et al. Comparison of 6-min walk test distance and cardiopulmonary exercise test performance in children with pulmonary hypertension. Arch Dis Child 2011; 96: 141-147.

23 Moledina S, Hislop AA, Foster H, et al. Childhood idiopathic pulmonary arterial hypertension: a national cohort study. Heart 2010; 96: 1401-1406.

24 Haworth SG, Hislop AA. Treatment and survival in children with pulmonary arterial hypertension: the UK Pulmonary Hypertension Service for Children 2001-2006. Heart 2009; 95: 312-317. 